\title{
Métodos para indução do parto
}

\author{
Methods for labor induction
}

\author{
Olímpio Barbosa de Moraes Filhoํ․ José Guilherme Cecatti², Francisco Edson de Lucena Feitosa ${ }^{3}$
}

\section{RESUM0}

O interesse da obstetrícia moderna pela indução do parto é demonstrado pela grande quantidade de artigos científicos publicados nos últimos anos. Os avanços da medicina em geral e da obstetrícia em particular têm permitido que mais gestações de risco evoluam até o termo ou próximo dele, com indicação materna ou fetal de interrupção da gestação antes do desencadeamento do trabalho de parto espontâneo. Isso coloca o obstetra na situação entre a escolha da cesárea ou da indução do parto. Para que o obstetra faça a escolha pela indução do parto e desta forma colabore com a diminuição da incidência de cesárea, é necessário que haja método acessível, barato, seguro, efetivo, de fácil utilização e de boa aceitabilidade. Embora exista grande quantidade de métodos de indução do parto relatados na literatura médica, sabe-se que ainda não há método ideal. No entanto, dentre eles, dois se destacam. O primeiro é a ocitocina, que possui as vantagens de promover contrações uterinas fisiológicas de trabalho de parto e com possibilidade de reverter os quadros de aumento da contratilidade uterina com a sua suspensão. O outro método é o misoprostol, o mais utilizado na atualidade, que amadurece o colo uterino e provoca contrações uterinas de trabalho de parto. No entanto, em relação ao misoprostol ainda existem controvérsias sobre sua dose e via ideal e segurança.

PALAVRAS-CHAVE: Trabalho de parto induzido/métodos; Gravidez

\section{ABSTRACT}

The interest of modern obstetrics in labor induction can be demonstrated by the huge amount of scientific articles published during the last few years. The advances of medicine in general and particularly of obstetrics allowed that more risky pregnancies reach term or near term, with a maternal or fetal indication for pregnancy interruption before the spontaneous onset of labor and delivery. This leads the obstetrician to the situation of choosing between cesarean section and labor induction. With the aim of helping the obstetrician to make the choice for labor induction and thus collaborate with the reduction in cesarean section rates, it is necessary that an accessible, cheap, safe, effective, easy to be used method with good acceptability is available. Although several methods of labor induction reported in medical literature do exist, it is known that there is no ideal method. However, among them, two are highlighted. The first is oxytocin, which has the advantages of promoting physiologic uterine contractions of labor and reverting uterine hypercontractility when suspended. The other method is misoprostol, nowadays the most used, which ripens the uterine cervix and induces uterine contractions of labor. However, there are still some controversies regarding its ideal dose, route and safety.

KEYWORDS: Labor, induced/methods; Pregnancy

\section{Introdução}

O termo "indução do parto" é utilizado para todos os procedimentos que podem provocar contrações uterinas e conseqüentemente o trabalho de parto em mulheres com mais de 22 semanas de idade gesta-

1 Professor Adjunto da Disciplina de Tocoginecologia da Faculdade de Ciências Médicas da Fundação Universidade de Pernambuco - UPE - Recife (PE), Brasil; Centro Integrado de Saúde Amaury de Medeiros (CISAM-UPE), Recife (PE), Brasil.

2 Professor Associado do Departamento de Tocoginecologia da Faculdade de Ciências Médicas da Universidade Estadual de Campinas - UNICAMP Campinas (SP), Brasil.

3 Coordenador da Clínica Obstétrica da Maternidade-Escola Assis Chateaubriand da Universidade Federal do Ceará - UFC - Fortaleza (CE), Brasil. Correspondência: Olímpio Barbosa de Moraes Filho

Rua 48, n 97, apto. 101 - Espinheiro - 52020-060 - Recife - PE - e-mail: olimpiomoraes@aol.com - Telefone: (81) 3427-2112 - Fax: (81) 3427-3513

Recebido em: 1/8/2005 Aceito com modificações em: 15/8/2005 
cional. O objetivo é promover o parto vaginal quando a continuação da gravidez significa risco materno-fetal maior do que a sua interrupção. A indução do parto, especialmente nas gestações de alto risco, representa estratégia importante para redução das taxas de cesariana, que aumentaram progressivamente nas duas últimas décadas na maioria dos países. No Brasil, essa ascensão fez com que a cesárea fosse considerada epidêmica pelas autoridades de saúde ${ }^{1}$.

Esse procedimento tem sido usado desde a sintese da ocitocina na década de 50. No entanto, principalmente nos últimos 10 anos, o conhecimento de novos métodos para indução do parto e a possibilidade de diagnóstico de complicações fetais cada vez mais preciso e precoce fizeram com que houvesse aumento vertiginoso de partos induzidos. Acredita-se que atualmente nos Estados Unidos em torno de $15 \%$ dos partos sejam induzidos ${ }^{2}$.

As principais indicações para indução do parto são: síndromes hipertensivas, gravidez prolongada, ruptura prematura de membranas, corioamnionite, diabetes, isoimunização fetal e restrição do crescimento fetal. As contra-indicações são praticamente as mesmas que contra-indicam o parto vaginal: macrossomia, apresentações anômalas, desproporção céfalo-pélvica, duas ou mais cicatrizes uterinas prévias, sofrimento fetal, placenta prévia completa e parcial, vasa prévia, prolapso de cordão umbilical, sorologia HIV+e herpes genital em atividade. Deve ser lembrado que antes de se tomar a decisão pela indução do parto, deve ser avaliada a vitalidade fetal com o uso da ultra-sonografia e/ou cardiotocografia ${ }^{3}$.

Um dos principais obstáculos ao êxito da indução é representado pelas condições cervicais desfavoráveis (índice de Bishop menor que 6). Baixos escores cervicais têm sido associados à falha de indução de trabalho de parto, ao seu prolongamento e ao elevado índice de cesariana ${ }^{4}$. Assim, métodos que permitem o amadurecimento cervical ao mesmo tempo em que estimulam a contratilidade uterina associam-se com taxas de sucesso mais elevadas.

Embora na atualidade esteja disponível arsenal muito mais amplo de alternativas de métodos de preparo cervical e indução do trabalho de parto, além de conhecimentos baseados em evidências científicas, tais procedimentos devem, sim, ser seriamente cogitados na atenção obstétrica como alternativa para a resolução do parto. Entretanto, devem ser empregados única e exclusivamente quando realmente é necessário que o parto ocorra antes de se desencadear espontaneamente, quando haja indicação clínica e/ou obstétrica para isso. Além do mais, só deve ser reali- zado em locais apropriados e com profissionais e instalações adequados para a necessária vigilância durante sua execução, para garantir a segurança, além da efetividade do método.

\section{Homeopatia}

O Caulophyllum thalictroides estaria indicado para regularizar as contrações uterinas no trabalho de parto, ou quando as contrações uterinas são fracas e irregulares, ou ainda na parada das contrações uterinas $^{5}$. Alguns homeopatas sugerem o uso de comprimido diariamente alguns dias antes da data provável do parto ou quando o trabalho de parto é iminente. Um ensaio clínico foi realizado para avaliar a efetividade do Caulophyllum antes do parto para tratamento de inércia uterina e na redução do risco de hemorragia pós-parto. Os autores concluíram que este remédio apresenta uma ação na prevenção de contrações uterinas fracas ${ }^{6}$. No entanto, atualmente são insuficientes as evidências para recomendar o uso de homeopatia na indução do parto ${ }^{7}$.

\section{Acupuntura}

Existem poucos estudos sobre acupuntura para indução do parto e a maioria é de descrição de séries de casos. Estes estudos sugerem que a acupuntura para indução do parto é método seguro, praticamente sem efeitos colaterais e que parece ser efetivo ${ }^{8-11}$. Apenas dois ensaios não randomizados foram realizados sobre o uso da acupuntura na indução do parto ${ }^{12,13}$. No primeiro ensaio clínico, usando eletroacupuntura, quatro eletrodos foram aplicados na pele do abdome para indução do parto no grupo de tratamento. O tratamento foi dado para 27 gestantes (77\%), comparadas com 102 gestantes sem intervenção. No grupo tratado, 20 gestantes pariram quatro dias antes da data provável, comparado com 47 (46\%) do grupo controle ${ }^{12}$. No segundo ensaio, a eletroacupuntura foi aplicada a 35 gestantes e outras 35 gestantes não receberam tratamento. Aumento de intensidade de contrações uterinas foi observado em 31 gestantes do grupo tratado, ao passo que no grupo controle não foi observado aumento da atividade uterina ${ }^{13}$.

Embora uma parcela importante da população prefira métodos alternativos, o uso da acupuntura na indução do parto deve ficar restrito a aqueles casos em que não há indicação médica para interrupção da gravidez. Ainda há a necessidade de ensaios clínicos bem controlados para avaliar o verdadeiro papel da acupuntura na indução do parto ${ }^{13}$.

\section{Estimulação mamária}

A estimulação mamária como método para indução do parto permite às gestantes um contro- 
le sobre o processo de indução e apresenta as vantagens de ser método natural e barato. Um estudo observacional demonstrou uma associação positiva entre a estimulação mamária (estimulação bilateral) e contração uterina ${ }^{14}$.

A estimulação mamária comparada com a não intervenção aumentou o número de parto dentro de 72 horas $(37,3$ versus $6,4 \%$ ) e diminuiu a taxa de hemorragia pós-parto $(0,7$ versus $6 \%)$. Porém, não houve diferenças entre os dois grupos quando o colo era desfavorável. Ainda são necessários mais estudos para indicar a estimulação mamária como método seguro e efetivo para indução do parto em gestantes de alto risco ${ }^{15}$.

\section{Relações sexuais}

A relação sexual poderia teoricamente induzir o parto por meio da estimulação física do segmento inferior uterino, ação direta das prostaglandinas do sêmen e/ou liberação endógena de ocitocina como resultado do orgasmo e estimulação dos mamilos. No entanto, não há ensaios clínicos bem controlados sobre o assunto ${ }^{16}$.

\section{Descolamento de membranas}

A técnica é relativamente simples: durante o toque vaginal, o dedo indicador do examinador é introduzido no canal cervical e, após identificar as membranas, realizam-se com a ponta do dedo movimentos circulares, com o intuito de descolar as membranas e dilatar o colo uterino. Este procedimento, além de uma ação mecânica sobre o colo uterino, promove também liberação de prostaglandinas $^{17}$.

Guando o descolamento de membranas é realizado de forma sistemática em gestantes a termo, observa-se redução na duração da gravidez e no número de gestantes que ultrapassam as 41 semanas, sem aumentar o risco materno ou neonatal de infecção. Os únicos efeitos adversos encontrados são de desconforto e sangramento genital durante ou logo após a realização do procedimento. No entanto, o uso rotineiro de descolamento de membranas na $38^{\mathrm{a}}$ semana não parece produzir benefícios clínicos importantes ${ }^{18}$.

\section{Métodos mecânicos}

Os métodos mecânicos nunca foram abandonados, mas vêm sendo substituídos pelos métodos farmacológicos nas últimas duas décadas. Baixo custo e redução de alguns efeitos colaterais são algumas vantagens que os métodos mecânicos apresentam sobre os métodos farmacológicos.

Um dos métodos mecânicos, descrito desde o século XVIII, é a laminária de alga marinha que, após ser submetida a um processo de desidratação, passava a ter propriedades hidrofílicas. Preparada sob a forma de bastão, era utilizada como dilatador cervical, mas seu uso foi abandonado pelo risco de infecção. Na década de 70 , com novas técnicas de esterilização, voltou a ser utilizada com alguns resultados satisfatórios. Seu mecanismo de ação depende do efeito mecânico, obtido pela sua expansão radial que, por ocorrer lentamente, não provoca lesão das fibras musculares do canal cervical ${ }^{19}$. Mais recentemente sua utilização clínica para esta finalidade tem sido preterida em favor de outros métodos mais efetivos.

O único método mecânico que continua sendo bastante utilizado é a sonda de Foley. Esta age não somente por meio da ação mecânica, mas também liberando prostaglandinas. A liberação de prostaglandinas seria conseqüência da separação do cório da decídua. Para que se obtenha sucesso na indução do parto com a utilização da sonda de Foley, geralmente é necessária a associação com ocitocina. Ou seja, a sonda promove o amadurecimento cervical, ao passo que a ocitocina é responsável pelo incremento da contração uterina. Desta maneira, sonda Foley e ocitocina podem ser tão efetivas quanto o misoprostol para indução do parto ${ }^{20-23}$. A associação da sonda de Foley com ocitocina diminui o risco de cesárea em relação ao parto que foi induzido apenas com a ocitocina ${ }^{24}$.

\section{Relaxina}

Relaxina é uma proteína composta por duas cadeias de aminoácidos. O seu papel durante a gravidez e parto ainda é pouco claro. Embora seu envolvimento no processo de preparo cervical no trabalho de parto seja considerado desde a década de 50, só recentemente, com advento das técnicas de DNA recombinante, a relaxina humana pôde ser sintetizada e melhor estudada. É possivel que a relaxina promova o amadurecimento cervical e, ao mesmo tempo, iniba as contrações uterinas. O uso da relaxina melhorou a condição do colo uterino sem causar hiperestimulação uterina, no entanto a taxa de cesárea foi igual entre as gestantes que receberam tratamento e naquelas que utilizaram placebo. São necessários mais ensaios clínicos para demonstrar o verdadeiro papel da relaxina na indução de parto $^{25}$.

\section{Mifepristona}

Nova classe de agentes farmacológicos tem sido desenvolvida para antagonizar a 
progesterona. O mais conhecido desses agentes é mifepristona, também chamado de RU486, que foi desenvolvido inicialmente para facilitar as técnicas utilizadas para realização do aborto. Alguns estudos mostram que a mifepristona pode induzir o parto ou amadurecer o colo uterino em gestações a termo ${ }^{26,27}$. Sete ensaios clínicos randomizados, envolvendo 594 gestantes, usando o mifepristona em diferentes doses, mostraram que após 48 e 96 horas, o colo desfavorável foi menos freqüente no grupo das gestantes tratadas. Também no grupo das gestantes que receberam mifepristona, o parto ocorreu com mais freqüência dentro do período de 48 e 96 horas do que no grupo que recebeu placebo. As gestantes tratadas com mifepristona tiveram menor número de cesárea. Não houve diferença entre os dois grupos em relação aos efeitos colaterais neonatais e maternos ${ }^{28}$.

No entanto, em recente ensaio clínico com 346 gestantes a termo com índice de Bishop igual ou menor que 4 , a indução do parto falhou com diferentes doses $(50,100,200,400$ até $600 \mathrm{mg})$ de mifepristona, mesmo esperando até 54 ho$\operatorname{ras}^{29}$

\section{Hialuronidase}

O mecanismo de ação da hialuronidase consiste, basicamente, em despolimerizar os componentes conjuntivos do colo uterino (colágeno, ácido hialurônico e condroitina), diminuindo a adesão celular do colágeno do colo uterino, proporcionando assim seu amolecimento e esvaecimento ${ }^{30}$.

A hialuronidase é considerada um método apenas para o preparo de colo, pois não promove o trabalho de parto. É de custo acessível e está disponivel no mercado nacional, podendo ser utilizada ambulatorialmente antes da internação para a indução. No entanto, revisão sistemática realizada pela Cochrane Library não encontrou ensaios clínicos sobre o assunto. Desta forma, o papel da hialuronidase como método de amadurecimento cervical e indução do parto permanece incerto ${ }^{31}$.

Recentemente, um ensaio clínico randomizado mostrou que a hialuronidase é menos efetiva do que a sonda de Foley em gestantes a termo. O grupo da hialuronidase apresentou maior tempo de indução, foi necessário maior dose de ocitocina adicional e foi menor o número de partos vaginais. No entanto, o conforto referido pelas pacientes foi mais freqüente no grupo da hialuronidase ${ }^{32}$.

\section{Ocitocina}

Desde que foi publicado o primeiro artigo sobre o uso contínuo e intravenoso da ocitocina em 1948, múltiplos esquemas para indução do parto foram apresentados por vários autores. Estes variam em relação à dose inicial, intervalo entre o aumento da dose e a quantidade da droga que é acrescida após cada intervalo. O ACOG (American College of Obstetricians and Gynecologists) orienta que a ocitocina deve ser iniciada com a dose de 1 a $2 \mathrm{mU} / \mathrm{min}$ e gradualmente acrescentada de 1 a $2 \mathrm{mU} / \mathrm{min}$, a cada 30 minutos, até desencadeamento do trabalho de parto $^{33}$. Outro esquema, utilizado por alguns autores, refere dose inicial de ocitocina de 1 a $2 \mathrm{mU} /$ min, duplicando a cada 30 ou 40 minutos, até o máximo de $16 \mathrm{mU} / \mathrm{min}$ (por exemplo, 1, 2, 4, 8 e $16 \mathrm{mU} / \mathrm{min})^{34}$.

A ação da ocitocina é dependente da presença de estrógenos que induzem aumento dos receptores para ocitocina no miométrio e apresentam níveis séricos tanto mais elevados, quanto mais avançada a idade gestacional. Em decorrência disto, a melhor resposta à ocitocina ocorre nas últimas semanas de gestação ${ }^{35}$.

Guando o colo uterino é imaturo, a indução do parto apenas com a utilização da ocitocina está associada a percentual elevado de partos prolongados, de doses elevadas com o risco de intoxicação hídrica, de falhas e, conseqüentemente, aumento da incidência de cesáreas ${ }^{1}$. Nesta situação, praticamente já é consenso que outros agentes, como o misoprostol, têm melhor efetividade ${ }^{36}$. Alguns autores chegam mesmo a desaconselhar completamente o uso isolado da ocitocina para induzir o parto quando o colo encontra-se imaturo $^{37}$.

Em revisão sistemática conclui-se que o parto vaginal ocorreu em $46 \%$ dos dentro das primeiras 24 horas quando se emprega a ocitocina contra 8,3\% no grupo de conduta expectante. Além disso, como mostra a Tabela 1 com os principais resultados desta revisão, o uso isolado da ocitocina comparada ao placebo ou conduta expectante também se associa com um aumento na proporção de partos por cesárea e uma diminuição em infecção neonatal ${ }^{38}$. No entanto, o uso isolado da ocitocina está associado a maior número de falhas de indução do parto que o uso da prostaglandina com a ocitocina (52 versus 28\%). O que se pode concluir, de forma generalizada, é que a ocitocina deve ser usada em conjunto com outro método ou agente que amadureça o colo uterino, aumentando desta forma a chance de sucesso da indução do parto ${ }^{38}$. 
Tabela 1 - Principais resultados de efetividade e segurança associados com o uso da ocitocina (versus placebo ou expectante) para a indução do trabalho de parto.

\begin{tabular}{lccc}
\hline Resultados & Ocitocina & $\begin{array}{c}\text { Placebol } \\
\text { expectante }\end{array}$ \\
\hline Sem parto vaginal em 24 h & $16 / 191$ & $112 / 208$ & $0,16(0,10-0,25)$ \\
Síndrome de hiperestimulação & $0 / 55$ & $2 / 45$ & $0,16(0,01-3,34)$ \\
Cesárea & $339 / 3267$ & $299 / 3353$ & $1,17(1,01-1,36)$ \\
Morbidade neonatal/ & $6 / 2373$ & $11 / 2433$ & $0,63(0,26-1,51)$ \\
mortalidade perinatal & & & \\
Hipercontratilidade & $4 / 1283$ & $2 / 1288$ & $2,01(0,37-10,94)$ \\
Rotura uterina & $0 / 1258$ & $1 / 2524$ & $0,67(0,03-16,40)$ \\
Parto instrumental & $430 / 2571$ & $415 / 2624$ & $1,05(0,93-1,18)$ \\
Mecônio & $91 / 1333$ & $110 / 1328$ & $0,83(0,64-1,08)$ \\
Apgar 50 min <7 & $28 / 2449$ & $46 / 2509$ & $0,68(0,44-1,07)$ \\
Admissão em UTI neonatal & $208 / 2196$ & $240 / 2191$ & $0,86(0,72-1,03)$ \\
Hemorragia pós-parto & $57 / 1303$ & $46 / 1308$ & $1,24(0,85-1,81)$ \\
Corioamnionite & $160 / 2720$ & $197 / 2795$ & $0,83(0,68-1,01)$ \\
Infecção neonatal & $39 / 2589$ & $63 / 2637$ & $0,65(0,44-0,95)$
\end{tabular}

Modificado de Kelly e Tan (2005) ${ }^{38}$. RR = risco relativo; IC 95\% = intervalo de confiança a $95 \%$.

\section{Dinoprostona}

Em 1992, a dinoprostona (prostaglandina $\mathrm{E}_{2}$ ) foi aprovada pela Food and Drug Administration (FDA) dos Estados Unidos, com o objetivo de promover o amadurecimento cervical $^{39}$. Já no Brasil, a dinoprostona nunca esteve disponivel comercialmente.

Para reduzir os inconvenientes das aplicações repetidas da prostaglandina $\mathrm{E}_{2}$ na endocérvice, foi desenvolvido pessário de silicone para uso intravaginal contendo $10 \mathrm{mg}$ de dinoprostona, que libera $0,3 \mathrm{mg} /$ hora do medicamento por até 12 horas, e que deve ser inserido no fundo de saco vaginal posterior. Esta apresentação permite a sua remoção quando desejado ou nos casos de hiperestimulação uterina ${ }^{40}$. No entanto, há limitações referentes ao elevado custo e à termolabilidade, o que dificulta a estocagem, além da necessidade freqüente do uso de ocitocina após o amadurecimento do colo uterino ${ }^{41}$. Foi, durante muito tempo, a droga padrão para o amadurecimento cervical e indução do trabalho de parto em países desenvolvidos, até o início da década de 90, quando o misoprostol, então mais conhecido e estudado, mostrou sua superioridade.

\section{Misoprostol}

Embora não previsto inicialmente como parte de suas indicações, o misoprostol (composto sintético análogo da prostaglandina $\mathrm{E}_{1}$ ) ganhou popularidade em obstetrícia, descrevendo-se inicialmente o uso para interrupção das gestações de pri- meiro trimestre, já em $1986^{42}$, e, logo em seguida, para interrupção da gravidez com feto morto ${ }^{43}$.

O misoprostol atua sobre a matriz extracelular, com dissolução das fibras colágenas, aumento do ácido hialurônico e aumento do conteúdo de água da cérvice. Além disso, relaxa o músculo liso da cérvice e facilita a dilatação, ao mesmo tempo em que permite o acréscimo do cálcio intracelular, promovendo contração uterina. Todos estes mecanismos permitem o progressivo esvaecimento e dilatação cervical, concomitante ao aumento da atividade uterina, o que garante na maior parte dos casos uma indução bem sucedida do trabalho de parto ${ }^{44}$.

Tanto a FEBRASGO (Federação Brasileira das Sociedades de Ginecologia e Obstetrícia), como o próprio Ministério da Saúde, vêm estimulando o uso do misoprostol nos centros obstétricos de referência, na dose de $25 \mu \mathrm{g}$ de seis em seis horas, em gestações a termo com feto vivo, para indução do trabalho de parto, com objetivo também de diminuir o número de cesáreas ${ }^{1}$.

O misoprostol apresenta importantes vantagens para o uso na clínica obstétrica. Seu custo é inferior ao de que qualquer outra prostaglandina; tem tempo de meia-vida alargado; é de fácil administração e não requer refrigeração para sua estocagem. Estudos comparativos mostraram que o misoprostol é tão eficiente ou mais que as outras prostaglandinas e mais eficaz que a ocitocina na indução do parto com colo imaturo ${ }^{36,45}$. Comparativamente à ocitocina, o misoprostol por via vaginal está associado a um maior sucesso da indução do trabalho de parto, embora também a um aumento da hipercontratilidade uterina, mas sem repercussões sobre a freqüência cardíaca fetal (Tabela 2) ${ }^{45}$.

Tabela 2 - Principais resultados de efetividade e segurança associados com o uso do misoprostol vaginal versus ocitocina para a indução do trabalho de parto.

\begin{tabular}{lccc}
\hline Resultados & $\begin{array}{c}\text { Misoprostol } \\
\text { vaginal }\end{array}$ & Ocitocina & RR (IC 95\%) \\
\hline Sem parto vaginal em 24 h & $75 / 262$ & $128 / 278$ & $0,66(0,4-1,00)$ \\
Síndrome de hiperestimulação & $42 / 437$ & $20 / 475$ & $1,72(0,69-4,25)$ \\
Cesárea & $159 / 866$ & $213 / 901$ & $0,82(0,59-1,15)$ \\
Hipercontratilidade & $178 / 620$ & $85 / 651$ & $2,22(1,77-2,79)$ \\
Rotura uterina & $2 / 17$ & $0 / 21$ & $6,11(0,31-119,34)$ \\
Parto instrumental & $55 / 479$ & $69 / 513$ & $0,84(0,49-1,43)$ \\
Mecônio & $58 / 420$ & $62 / 454$ & $1,02(0,73-1,41)$ \\
Apgar 50 min <7 & $16 / 614$ & $13 / 643$ & $1,26(0,62-2,53)$ \\
Admissão em UTI neonatal & $59 / 376$ & $60 / 408$ & $1,04(0,76-1,44)$ \\
Hemorragia pós-parto & $4 / 121$ & $8 / 124$ & $0,51(0,16-1,66)$
\end{tabular}

Modificada de Hofmeyr e Gülmezoglu (2005) ${ }^{45}$. RR = risco relativo; IC 95\% = intervalo de confiança a 95\%. 
O misoprostol está associado à baixa morbimortalidade perinatal sendo neste aspecto, semelhante aos outros métodos de indução. Quando se utiliza a dose recomendada de $25 \mu \mathrm{g}$ de 6 em 6 horas, a possibilidade de alterações da contratilidade uterina e de sindrome da hiperestimulação é baixa, ficando em torno dos $7 \%$ e 3\%, respectivamente ${ }^{46}$.

O risco de eliminação de mecônio intraparto parece aumentado quando o misoprostol é utilizado, em comparação com outros métodos, porém os desfechos neonatais não são afetados ${ }^{45}$. A possibilidade de mecônio aumenta de acordo com o aumento da dose utilizada. Na dose de $50 \mu \mathrm{g}$ de quatro em quatro horas, a incidência de mecônio é de $21,5 \%{ }^{21}$, muito superior aos $12 \%$ esperado no trabalho de parto espontâneo. No entanto, na dose recomendada atualmente, não tem sido observado aumento da incidência de mecônio ${ }^{46}$. De qualquer forma, a preocupação com eliminação de mecônio deve estar presente quando se acompanha o trabalho de parto induzido por misoprostol.

Em revisão da literatura, focalizando gestantes com cesárea anterior submetidas à indução do parto com doses maiores de misoprostol que as que são utilizadas hoje, foi observada incidência de $5,6 \%$ de ruptura uterina ${ }^{47}$. Nenhum dos estudos utilizados para esta revisão foi desenhado especificamente com esse objetivo. Assim, o poder da amostra pode ter sido insuficiente para determinar a real freqüência deste evento. Mesmo assim, baseando-se nesta revisão, o ACOG passou a desaconselhar a utilização de misoprostol para indução do parto em mulheres com cesárea anterior ou cicatriz uterina ${ }^{48}$.

Em estudo recente com 3.533 mulheres com passado de uma cesárea e no qual o parto foi induzindo houve ocorrência de ruptura uterina em $0,8 \%$ dos casos em que se usou ocitocina e em $1,1 \%$ dos com misoprostol. A indução do parto com ocitocina ou misoprostol está associada com aumento de ruptura uterina quando comparada com o trabalho de parto espontâneo. No entanto, entre as gestantes com passado de uma cesárea, não houve diferença significativa no número de rupturas uterinas entre as induzidas com misoprostol ou com ocitocina ${ }^{49}$.

O Brasil foi o primeiro país do mundo a disponibilizar comercialmente o misoprostol preparado especificamente para uso vaginal, na forma de compridos vaginais de $25 \mu \mathrm{g}$. Este preparado mostrou efetividade e segurança similares a doses equivalentes de misoprostol obtidas do fracionamento de comprimidos de $200 \mu \mathrm{g}$ comercializados para utilização por via oral $^{50}$. Sem dúvida a via vaginal para o uso de misoprostol é a mais estudada e utilizada. No entanto, vários estudos já foram publicados utilizando outras vias para o uso do misoprostol na indução do parto.

Em relação à via oral, embora não haja diferença em relação a efeitos adversos, a dose necessária para induzir um parto é três a quatro vezes maior do que a dose utilizada por via vaginal $^{39}$. Este resultado já era esperado, pelo motivo da biodisponibilidade do misoprostol, por via vaginal, ser três vezes maior que por via oral ${ }^{51,52}$. A via oral para uso de misoprostol é via efetiva para indução do parto no terceiro trimestre. Porém, faltam dados para afirmar qual é o esquema posológico mais efetivo e seguro ${ }^{53}$.

Outra via que mais recentemente começou a ser avaliada em estudos é a via sublingual. Além de apresentar boa aceitabilidade, esta via apresenta resultados semelhantes à via vaginal em relação à efetividade, quando comparada com a mesma dose ${ }^{46}$. Mas ainda não há dados para se chegar a uma conclusão sobre as complicações e efeitos colaterais do misoprostol por via sublingual na indução do parto ${ }^{54}$.

Por último, outra via possivel de uso é a retal. O estudo da farmacocinética do misoprostol por via retal mostra um comportamento dos níveis séricos muito parecido com o que ocorre na via vaginal ${ }^{55}$. Recentemente um estudo nacional usou o misoprostol retal para indução do parto nos casos de ruptura prematura das membranas na dose de $50 \mathrm{mg}$ a cada 4 horas, com resultado de $72 \%$ de partos vaginais ${ }^{56}$. As biodisponibilidades do misoprostol por via retal e vaginal são maiores que por via oral, provavelmente pelo mesmo motivo, que é de evitar a primeira passagem hepática ${ }^{57}$.

\section{Referências}

1. Ministério da Saúde. Secretaria de Políticas de Saúde. Área Técnica de Saúde da Mulher. Parto, aborto e puerpério: assistência humanizada à mulher. Brasília (DF): Ministério da Saúde; 2001. p. 32-7.

2. American College of Obstetricians and Gynecologists-ACOG. Committee Opinion. Induction of labor with misoprostol. Int $\mathrm{J}$ Gynaecol Obstet. 2000;69(1):77-8.

3. Cecatti JG, Parpinelli MA. Indução do parto. In: Guariento A, Mamed JAV, editores. Medicina materno-fetal. São Paulo: Atheneu; 2001. p. 143952.

4. Rouse DJ, Owen J, Hauth JC. Criteria for failed labor induction: prospective evaluation of a standardized protocol. Obstet Gynecol. 2000;96(5 Pt 1):671-7. 
5. Priestman KG. A few useful remedies in pregnancy, labour and the first few days of the babies' life. $\mathrm{Br}$ Homeopath J. 1988;77(3):172-3.

6. Smith CA. Homoeopathy for induction of labour. Cochrane Database Syst Rev. 2005;(2):CD003399.

7. Ventoskovskiy BM, Popov AV. Homeopathy as a practical alternative to traditional obstetric methods. Br Homeopath J. 1990;79(4):201-5.

8. Tsuei JJ, Lai YF. Induction of labor by acupuncture and electrical stimulation. Obstet Gynecol. 1974;43(3):337-42.

9. Yip SK, Pang JC, Sung ML. Induction of labor by acupuncture electro stimulation. Am J Chin Med (Gard City N Y). 1976;4(3):257-65.

10.Tsuei JJ, Lai Y, Sharma SD. The influence of acupuncture stimulation during pregnancy: the induction and inhibition of labor. Obstet Gynecol. 1977;50(4):479-88.

11. Theobald GW. The electrical induction of labour. New York: Appleton-Century-Crofts; 1973.

12. Kubista E, Kucera H, Muller-Tyl E. Initiating contractions of the gravid uterus through electroacupuncture. Am J Chin Med (Gard City N Y). 1975;3(4):343-6.

13. Smith CA, Crowther CA. Acupuncture for induction of labour. Cochrane Database Syst Rev. 2005;(2):CD002962.

14. Lenke RR, Nemes JM. Use of nipple stimulation to obtain contraction stress test. Obstet Gynecol. 1984;63(3):345-8.

15. Kavanagh J, Kelly AJ, Thomas J. Breast stimulation for cervical ripening and induction of labour. Cochrane Database Syst Rev. 2005;(3):CD003392.

16. Kavanagh J, Kelly AJ, Thomas J. Sexual intercourse for cervical ripening and induction of labour. Cochrane Database Syst Rev. 2005;(2):CD003093.

17. Keirse MJ, Thiery M, Parewijck W, Mitchell MD. Chronic stimulation of uterine prostaglandin synthesis during cervical ripening before the onset of labor. Prostaglandins. 1983;25(5):671-82.

18. Boulvain M, Stan C, Irion O. Membrane sweeping for induction of labour. Cochrane Database Syst Rev. 2005;(1):CD000451.

19. Parpinelli MA, Cecatti JG, Ribeiro ST, Pires HMB, Faúndes A. Uso da laminária no preparo do colo uterino para indução do parto em gestações com óbito fetal. Rev Bras Ginecol Obstet. 1996;18(9):6936.

20. Sciscione AC, McCullough H, Manley JS, Shlossman PA, Pollock M, Colmorgen GH. A prospective randomized comparison of Foley catheter insertion versus intracervical prostaglandin $\mathrm{E}_{2}$ gel for preinduction cervical ripening. Am J Obstet Gynecol. 1999; 180(1 Pt 1):55-60.
21. Sciscione AC, Nguyen L, Manley J, Pollock M, Maas B, Colmorgen G. A randomized comparison of transcervical Foley catheter to intravaginal misoprostol for preinduction cervical ripening. Obstet Gynecol. 2001;97(4):603-7.

22. Upadhyaya NB, Childs KD, Neiger R, Caudle MR. Ambulatory cervical ripening in term pregnancy. $\mathrm{J}$ Reprod Med. 1999;44(4):363-6.

23. Sciscione AC, Muench M, Pollock M, Jenkins TM, Tildon-Burton J, Colmorgen GH. Transcervical Foley catheter for preinduction cervical ripening in an outpatient versus inpatient setting. Obstet Gynecol. 2001;98(5 Pt 1):751-6.

24. Boulvain M, Kelly A, Lohse C, Stan C, Irion O. Mechanical methods for induction of labour. Cochrane Database Syst Rev. 2005;(2):CD001233.

25. Kelly AJ, Kavanagh J, Thomas J. Relaxin for cervical ripening and induction of labour. Cochrane Database Syst Rev. 2005;(2):CD003103.

26. Chwalisz K. The use of progesterone antagonists for cervical ripening and as an adjunct to labour and delivery. Hum Reprod. 1994;9 Suppl 1:131-61.

27. Stenlund PM, Ekman G, Aedo AR, Bygdeman M. Induction of labor with mifepristone: a randomized, double-blind study versus placebo. Acta Obstet Gynecol Scand. 1999;78(9):793-8.

28. Neilson JP. Mifepristone for induction of labour. Cochrane Database Syst Rev. 2005;(2):CD002865.

29. Berkane N, Verstraete L Uzan S, Boog G, Maria B. Use of mifepristone to ripen the cervix and induce labor in term pregnancies. Am J Obstet Gynecol. 2005; 192(1):114-20.

30. Spallici MDB, Chiea MA, Abuquerque PB, Singer JM, Bittar RE, Zugaib M. Ação da hialuronidase na maturação do colo uterino em gestações a termo. Rev Ginecol Obstet. 2000;11(2):93-102.

31. Kavanagh J, Kelly AJ, Thomas J. Hyaluronidase for cervical priming and induction of labor. Cochrane Database Syst Rev. 2005;(2):CD003097.

32. Surita FG, Cecatti JG, Parpinelli MA, Krupa JL, Pinto e Silva JL. Hyaluronidase versus Foley catheter for cervical ripening in high-risk term and post term pregnancies. Int J Gynaecol Obstet. 2005;88(3):25864.

33.American College of Obstetricians and Gynecologists-ACOG. Induction and augmentation of labor. Washington (DC): ACOG; 1991. (Technical Bulletin, 157).

34. Blanco JD, Finley BE. Induction and simulation of labor. In: Pauerstein GJ, editor. Clinical obstetrics. New York: John Wiley; 1987. p. 495-501.

35. Satin AJ, Leveno KJ, Sherman ML, McIntire DD. Factors affecting the dose response to oxytocin for labor stimulation. Am J Obstet Gynecol. 1992;166(4):1260-1. 
36. Aquino MM, Cecatti JG. Misoprostol versus oxytocin for labor induction in term and post-term pregnancy: randomized controlled trial. Sao Paulo Med J. 2003; 121(3):102-6.

37. den Hertog $\mathrm{CE}$, de Groot AN, van Dongen PW. History and use of oxytocics. Eur J Obstet Gynecol Reprod Biol. 2001;94(1):8-12.

38. Kelly AJ, Tan B. Intravenous oxytocin alone for cervical ripening and induction of labour. Cochrane Database Syst Rev. 2005;(2):CD003246.

39. Wing DA, Ham D, Paul RH. A comparison of orally administered misoprostol with vaginally administered misoprostol for cervical ripening and labor induction. Am J Obstet Gynecol. 1999; 180(5):1155-60.

40. Rayburn WF, Wapner RJ, Barss VA, Spitzberg E, Molina $\mathrm{RD}$, Mandsager $\mathrm{N}$, et al. An intravaginal controlled-release prostaglandin $\mathrm{E}_{2}$ pessary for cervical ripening and initiation of labor at term. Obstet Gynecol. 1992;79(3):374-9.

41. Buser D, Mora G, Arias F. A randomized comparison between misoprostol and dinoprostone for cervical ripening and labor induction in patients with unfavorable cervices. Obstet Gynecol. 1997;89(4):581-5.

42. Herting RL, Nissen CH. Overview of misoprostol clinical experience. Dig Dis Sci. 1986;31(2 Suppl):47S-54S.

43. Mariani Neto C, Leão EJ, Barreto EMCP, Kenj G, Aquino MMA, Tuffi VHB. Uso do misoprostol para indução do parto com feto morto. Rev Paul Med. 1987;105(6):325-8.

44. Arias F. Pharmacology of oxytocin and prostaglandins. Clin Obstet Gynecol. 2000;43(3):455-68.

45. Hofmeyr GJ, Gülmezoglu AM. Vaginal misoprostol for cervical ripening and induction of labour. Cochrane Database Syst Rev. 2005;(2):CD000941.

46. Moraes Filho OB, Albuquerque RM, Pacheco AJC, Ribeiro RH, Cecatti JG, Welkovic S. Misoprostol sublingual versus vaginal para indução do parto a termo. Rev Bras Ginecol Obstet. 2005;27(1):24-31.

47. Plaut MM, Schwartz ML, Lubarsky SL. Uterine rupture associated with the use of misoprostol in the gravid patient with a previous cesarean section. Am J Obstet Gynecol. 1999;180(6 Pt 1):1535-42.
48. American College of Obstetricians and Gynecologists-ACOG. Induction of labor with misoprostol. Washington (DC): ACOG; 1999.

49. Lin C, Raynor BD. Risk of uterine rupture in labor induction of patients with prior cesarean section: an inner city hospital experience. Am J Obstet Gynecol. 2004;190(5):1476-8.

50. Cecatti JG, Tedesco RP, Besteti Pires HM, Calderón IM, Faúndes A. Effectiveness and safety of a new vaginal misoprostol product specifically labeled for cervical ripening and labor induction. Acta Obstet Ginecol Scand. In press 2005.

51.Zieman M, Fong SK, Benowitz NL, Banskter D, Darney PD. Absorption kinetics of misoprostol with oral or vaginal administration. Obstet Gynecol. 1997;90(1):88-92.

52. Tang OS, Schweer H, Seyberth HW, Lee SW, HO PC. Pharmacokinetics of different routes of administration of misoprostol. Hum Reprod. 2002;17(2):332-6.

53. Alfirevic Z. Oral misoprostol for induction of labour. Cochrane Database Syst Rev. 2005;(2):CD001338.

54.Muzonzini G, Hofmeyr GJ. Buccal or sublingual misoprostol for cervical ripening and induction of labour. Cochrane Database Syst Rev. 2005;(2):CD004221.

55. Walraven G, Dampha Y, Bittaye B, Sowe M, Hofmeyr $\mathrm{J}$. Misoprostol in the treatment of postpartum haemorrhage in addition to routine management: a placebo randomised controlled trial. BJOG. 2004;111(9):1014-7.

56. Arcanjo FCN, Alencar Júnior CA, Feitosa FEL, Amorim MMR. Uso de misoprostol retal para indução do parto em gestantes com amniorrexe prematura: ensaio clínico fase II. Rev Bras Ginecol Obstet. 2003;25(7):491-9.

57. Khan RU, El-Refaey H. Pharmacokinetics and adverse-effect profile of rectally administered misoprostol in the third stage of labor. Obstet Gynecol. 2003;101(5 Pt 1):968-74. 